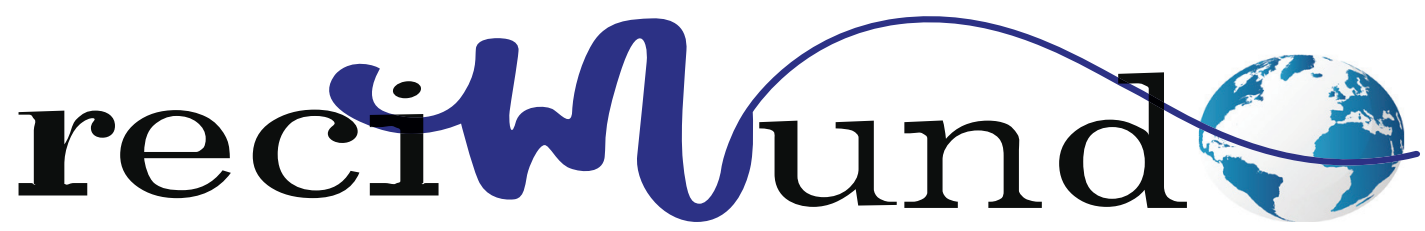

Revista Científica Mundo de la Investigación y el Conocimiento

DOI: 10.26820/recimundo/5.(4).oct.2021.143-151

URL: https://recimundo.com/index.php/es/article/view/1312

EDITORIAL: Saberes del Conocimiento

REVISTA: RECIMUNDO

ISSN: 2588-073X

TIPO DE INVESTIGACIÓN: Artículo de revisión

CÓDIGO UNESCO: 32 Ciencias Médicas

PAGINAS: $143-151$

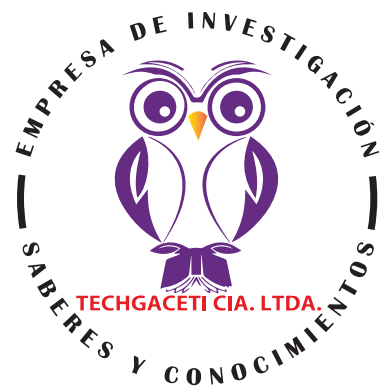

\title{
Niveles de Parathormona relacionado con eventos coronarios agudos en pacientes con enfermedad renal crónica
}

Parathormone levels related to acute coronary events in patients with chronic kidney disease

Níveis de paratormônio relacionados a eventos coronários agudos em pacientes com doença renal crônica

Carolina Estefanía Lema Tixi; Eduardo Patricio Padilla Manzano; Ángelo Rigoberto Salinas Martínez

RECIBIDO: 02/09/2021 ACEPTADO: 20/09/2021 PUBLICADO: 30/10/2021

1. Médico de la Universidad de Guayaquil; Médico Postgradista R2 Especialidad Cardiología; Guayaquil, Ecuador; md.carolinalema@hotmail.com; (iD https://orcid.org/0000-0001-9580-2097

2. Médico de la Universidad de Guayaquil; Médico Postgradista R2 Especialidad Medicina Interna; Guayaquil, Ecuador; eduardoppm17@hotmail.com; (D) https://orcid.org/0000-0003-3646-4117

3. Médico de la Universidad de Guayaquil; Investigador Independiente; Guayaquil, Ecuador; angelosalinasm@hotmail.com; (iD) https://orcid.org/0000-0003-2210-8429

CORRESPONDENCIA

Carolina Estefanía Lema Tixi

md.carolinalema@hotmail.com

Guayaquil, Ecuador

C RECIMUNDO; Editorial Saberes del Conocimiento, 2021 


\title{
RESUMEN
}

La enfermedad coronaria es una de las complicaciones más frecuentes en los pacientes con enfermedad renal crónica, debido en parte a la alta incidencia de factores de riesgo comunes, como la hipertensión arterial o la diabetes mellitus, y a factores dependientes de la propia enfermedad renal, como lo es el hiperparatiroidismo. Diversos estudios han demostrado la existencia de una relación entre un mayor riesgo cardiovascular y la presencia de hiperparatiroidismo, bien sea este primario o secundario. Dicha relación parece atribuible, a un aumento de la prevalencia de los factores de riesgo cardiovascular entre aquellos pacientes con hiperparatiroidismo primario asintomático. La elevación de la hormona paratiroidea se relaciona con el desarrollo de eventos coronarios agudos en pacientes con nefropatía sometidos a hemodiálisis y, el valor de corte de $500 \mathrm{pg} / \mathrm{dl}$ o más, hace que sea frecuente la aparición de complicaciones cardiovasculares. El enfoque general del presente trabajo es dar a conocer la incidencia de los Niveles de Parathormona en la aparición de eventos coronarios agudos en pacientes con enfermedad renal crónica. Los resultados se obtuvieron mediante el desarrollo de una investigación de tipo bibliográfica, delimitada a una metodología de revisión, que permite concluir que el hecho de tener niveles de Paratohormona (PTH) elevados, resulta ser un factor de riesgo importante para la aparición de eventos coronarios agudos, sin embargo, todavía es muy pobre la evidencia en lo que respecta a la relación existente entre los niveles de PTH y el pronóstico una vez la enfermedad coronaria está establecida, por lo que se recomienda realizar estudios prospectivos que permitan ampliar los hallazgos encontrados en la presente investigación.

Palabras clave: Paratohormona, paratiroidea, coronario, renal, hormona, hiperparatiroidismo.

\begin{abstract}
Coronary heart disease is one of the most frequent complications in patients with chronic kidney disease, due in part to the high incidence of common risk factors, such as high blood pressure or diabetes mellitus, and factors dependent on the kidney disease itself, such as it is hyperparathyroidism. Several studies have shown the existence of a relationship between an increased cardiovascular risk and the presence of hyperparathyroidism, whether it is primary or secondary. This relationship seems to be attributable to an increase in the prevalence of cardiovascular risk factors among those patients with asymptomatic primary hyperparathyroidism. The elevation of parathyroid hormone is related to the development of acute coronary events in patients with nephropathy undergoing hemodialysis, and the cut-off value of $500 \mathrm{pg} / \mathrm{dl}$ or more makes the appearance of cardiovascular complications frequent. The general approach of this work is to show the incidence of Parathormone Levels in the appearance of acute coronary events in patients with chronic kidney disease. The results were obtained through the development of a bibliographic research, limited to a review methodology, which allows to conclude that having elevated Parathyroid hormone (PTH) levels turns out to be an important risk factor for the appearance of coronary events However, the evidence regarding the relationship between PTH levels and prognosis once coronary artery disease is established is still very poor, so prospective studies are recommended to expand the findings found in the present investigation.
\end{abstract}

Keywords: Parathyroid hormone, parathyroid, coronary, renal, hormone, hyperparathyroidism.

\section{RESUMO}

A doença coronariana é uma das complicações mais frequentes em pacientes com doença renal crônica, em parte devido à alta incidência de fatores de risco comuns, como hipertensão ou diabetes mellitus, e de fatores dependentes da própria doença renal, como é hiperparatireoidismo. Vários estudos têm demonstrado a existência de relação entre o aumento do risco cardiovascular e a presença de hiperparatireoidismo, seja ele primário ou secundário. Essa relação parece ser atribuível a um aumento na prevalência de fatores de risco cardiovascular entre aqueles pacientes com hiperparatireoidismo primário assintomático. A elevação do paratormônio está relacionada ao desenvolvimento de eventos coronários agudos em pacientes com nefropatia em hemodiálise, e o valor de corte de $500 \mathrm{pg} / \mathrm{dl}$ ou mais torna frequente o aparecimento de complicações cardiovasculares. A abordagem geral deste trabalho é mostrar a incidência dos Níveis de Paratormônio no aparecimento de eventos coronários agudos em pacientes com doença renal crônica. Os resultados foram obtidos por meio do desenvolvimento de uma pesquisa bibliográfica, limitada a uma metodologia de revisão, que permite concluir que ter níveis elevados de hormônio da paratireóide (PTH) acaba sendo um importante fator de risco para o surgimento de eventos coronarianos. a relação entre os níveis de PTH e o prognóstico após o estabelecimento da doença arterial coronariana ainda é muito pobre, de modo que estudos prospectivos são recomendados para expandir os achados encontrados na presente investigação.

Palavras-chave: Hormônio da paratireóide, paratireóide, coronário, renal, hormônio, hiperparatireoidismo. 


\section{Introducción}

Las enfermedades cardiovasculares (ECV) constituyen la primera causa de morbi-mortalidad a nivel mundial, con un aproximado de 17,5 millones de decesos por año. Se caracterizan por ser de origen multifactorial, donde interviene muchos factores de riesgos, entre los que destaca la hipertensión arterial. (Rojas et al. 2017).

Según lo indica Serje et al. (2014) la enfermedad coronaria es una de las complicaciones más frecuentes en los pacientes con nefropatía crónica, debido en parte a la alta incidencia de factores de riesgo comunes, como la hipertensión arterial o la diabetes mellitus, y a factores dependientes de la propia enfermedad renal, como lo es el hiperparatiroidismo.

La incidencia de los grandes síndromes cardíacos como la insuficiencia cardíaca o la cardiopatía isquémica de origen renal, es elevada y conlleva una alta tasa de morbimortalidad. La elevación de la hormona paratiroidea se relaciona con el desarrollo de eventos coronarios agudos en los pacientes con nefropatía sometidos a hemodiálisis y, el valor de corte de 500 pg/dl o más, hace que sea frecuente la aparición de complicaciones cardiovasculares. ( $p$. 15-22).

La hormona paratiroidea, también conocida como Paratohormona (PTH) es producida por las glándulas paratiroides, cuatro glándulas del tamaño de una arveja o chícharo ubicadas en el cuello. La PTH controla el nivel de calcio en la sangre. También es esencial para el buen funcionamiento de los nervios, los músculos y el corazón. (Medline Plus, 2020).

En ese mismo sentido Ramos et al. (2017) señala que se ha relacionado la presencia de niveles elevados de Paratohormona (PTH) con una enfermedad coronaria más compleja, e incluso con la presencia de mayor número de eventos adversos durante el seguimiento en pacientes diagnosticados de enfermedad coronaria estable.

La prevalencia de los niveles elevados de paratohormona entre los pacientes ingresados por Síndrome coronario agudo (SCA) fue elevada y su presencia se asoció con un perfil clínico más adverso y una peor evolución. (p. 73-81).

El objetivo de este trabajo investigativo, desarrollado bajo un diseño documental, se centra en realizar una revisión a la literatura científica disponible, que principalmente aborde a los niveles de Parathormona relacionado con eventos coronarios agudos en pacientes con enfermedad renal crónica, sus definiciones y principales factores de riesgo, todo ello con la finalidad de proporcionar un material actualizado que defina dichos aspectos y que sea de utilidad no solo para el personal de salud, sino también para el público en general.

\section{Materiales y Métodos}

El presente trabajo de investigación, enmarcado en una metodología de revisión, está orientado a la construcción de un material bibliográfico actualizado, enfocado en dar a conocer los criterios vigentes de los Niveles de Parathormona relacionado con eventos coronarios agudos en pacientes con enfermedad renal crónica.

Entre las bases de datos consultadas destacan: Organización Panamericana de la Salud (PAHO), Organización Mundial de la Salud (OMS), medigraphic, mayo clinic, entre otras. Como términos de búsqueda se utilizaron las expresiones "Niveles de Parathormona", "Parathormona", "eventos coronarios agudos", "enfermedad renal crónica", "eventos coronarios agudos y enfermedad crónica" y se aplicaron criterios de selección tales como: idioma español; publicación entre 2010 Y 2021 (ambos inclusive); acceso completo y abierto; en el

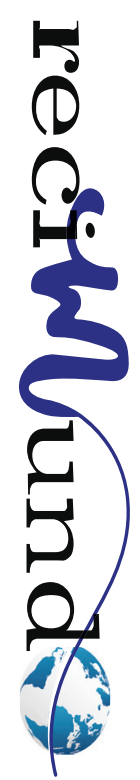


área de salud y medicina; estudios referidos a humanos; tipo de bibliografía, manuales médicos, guías clínicas, ensayos clínicos, estudios o reportes de casos, boletines y/o folletos de instituciones oficiales o privadas de excelente trayectoria en el área de la salud, medicina o científico académica, y demás, monografías y otros documentos que, a criterio propio, mostraran información de interés en base a la observación de la evidencia científica referida en sus contenidos. Este proceso arrojó resultados que en promedio oscilaron entre 9 y 62 enlaces a fuentes de información bibliográficas.

De igual manera fueron adelantados otras sondeos menores sin considerar la aplicación de cualquier otro criterio de descarte, ya que se requirió encontrar información complementaria que independientemente de su origen o época de publicación, es considerada igualmente importante ajustada y de relevancia para este tema. Es a partir de entonces que se procedió con la lectura crítica y análisis interpretativo de un pilar de información recopilado a lo largo de la investigación, que también fue adoptada como evidencia. Resultando todo este proceso en la selección de los elementos más sustanciales y significativos de las diferentes fuentes bibliográficas que fundamentan el razonamiento aquí expuesto.

\section{Resultados}

A pesar del desarrollo tecnológico, el avance de la medicina y el enriquecimiento de los conocimientos académicos de las diferentes sociedades, los eventos coronarios agudos continúan afectando a la población mundial, sin distinción de género, raza o condición económica. Hoy día son considerados la primera causa de mortalidad en muchos países. (Cediel et al. 2015).

Los síndromes coronarios agudos son el resultado de una obstrucción aguda de una arteria coronaria. Las consecuencias dependen del grado y la localización de la obstrucción y van desde angina inestable hasta infarto de miocardio sin supra desnivel del segmento ST, infarto de miocardio con elevación del segmento ST (IMEST) y muerte súbita de origen cardíaco. (Sweis \& Jivan, 2020).

Producto del seguimiento de múltiples grupos poblacionales a largo plazo, se ha identificado una serie de factores de riesgo para el desarrollo de la enfermedad cardiovascular. A este respecto, Cediel et al. (2015) Indica que:

Cobra vital importancia la pérdida progresiva del funcionamiento renal, situación que a su vez, se concatena con una serie de alteraciones fisiopatológicas que perpetúan el daño cardiovascular y aumentan el riesgo de enfermedad arterial coronaria aterosclerótica. Cerca del 10\% de la población mundial padece Enfermedad Renal Crónica (ERC), cifra que alcanza el $50 \%$ en la población geriátrica. Este hecho ha motivado un creciente interés por parte de las autoridades que han permitido el establecimiento de políticas preventivas y de vigilancia para todos los componentes de este evento. (p. 99-106). 


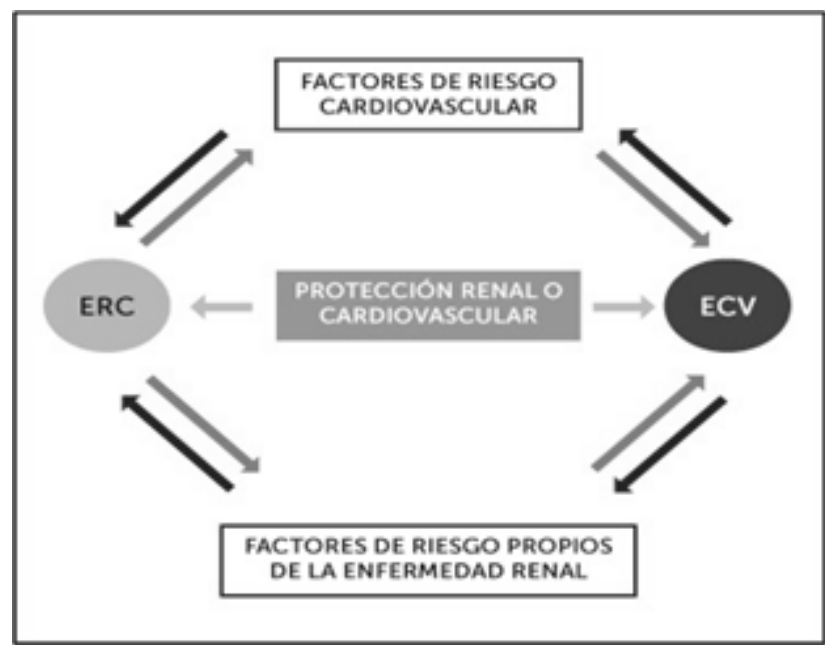

Figura 1. Interacciones cruzadas entre la enfermedad renal crónica (ERC) y la enfermedad cardiovascular (ECV).

Fuente: Enfermedad Cardiovascular (ECV) en la Enfermedad Renal Crónica (ERC). Orozco. (2015). Revista Médica Clínica Las Condes 26(2). Pág. 142-155.

Recuperado de: https://www.sciencedirect.com/science/article/pii/ S0716864015000358

La Enfermedad Renal Crónica (ERC), también llamada insuficiencia renal crónica, consiste en la pérdida gradual de la función renal. Los riñones filtran los desechos y el exceso de líquido de la sangre, que luego se excretan con la orina. Cuando llega a una etapa avanzada, pueden acumularse niveles peligrosos de líquidos, electrolitos y desechos en el cuerpo. (Mayo clinic, 2021)

La Organización Panamericana de la Salud/ Organización Mundial de la Salud (OPS/ OMS) (2014) señalan que la diabetes y la hipertensión, sumadas al envejecimiento, son los principales factores de riesgo para desarrollar la enfermedad renal crónica (ERC), que afecta a uno de cada diez adultos en el mundo. Y la definen como la pérdida progresiva de la función renal en meses o en años.
Entre las complicaciones de esta patología Mayo Clinic (2021) destaca las siguientes:

- Aumento repentino en los niveles de potasio en sangre (hiperpotasemia), lo cual puede alterar el funcionamiento del corazón.

- Enfermedad cardíaca y de los vasos sanguíneos (cardiovascular).

- Daño en el sistema nervioso central, lo cual causa dificultades de concentración, cambios de personalidad o convulsiones.

- Disminución en la respuesta inmunológica, lo cual lo hace más vulnerable a las infecciones.

- Pericarditis, una inflamación en la membrana en forma de saco que cubre el corazón (pericardio).

- Daño irreversible en los riñones (enfermedad renal terminal); eventualmente, necesitarás diálisis o un trasplante de riñón para sobrevivir.

Desde la primera descripción de la asociación entre enfermedad renal crónica (ERC) y enfermedad cardiovascular (ECV), Ios estudios epidemiológicos han confirmado y extendido el conocimiento acerca de esta relación. El riesgo de ECV se incrementa al progresar la falla renal, probablemente por la suma de los factores de riesgo no tradicionales propios de la insuficiencia renal. (Orozco, 2015).

La prevención de eventos cardiovasculares, en esta población, debe basarse en dos premisas fundamentales. La primera es iniciar el tratamiento cardiovascular en etapas tempranas de ERC. De allí la importancia en pesquisar la ERC en pacientes de riesgo, mediante la estimación de la Velocidad de Filtrado Glomerular Estimada (VFGe) y la medición de la albuminuria. Lo segundo es, en pacientes ya con una ERC más avanzada, implementar un abordaje multifactorial. Este enfoque terapéutico multimodal debe incluir educación, cambios en el estilo de vida, control de la glicemia, tratamiento de la hipertensión ar-

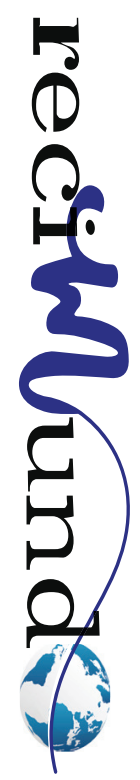


terial, control de los lípidos y uso de fármacos específicos entre otras medidas. ( $p$. 142-155).

Estudios previos han demostrado la existencia de una relación entre un mayor riesgo cardiovascular y la presencia de hiperparatiroidismo, bien sea este primario o secundario. Dicha relación parece atribuible, en gran medida, a un aumento de la prevalencia de los factores de riesgo cardiovascular entre aquellos pacientes con hiperparatiroidismo primario asintomático. (Ramos et al. 2017).

Por su parte Rojas et al. (2017) Coincide en que entre las causas de eventos coronarios agudos, el hiperparatiroidismo, particularmente en su variante primaria, recientemente se ha visto rodeado de controversia en aspectos epidemiológicos y bioquímicos.

La secreción excesiva de hormona paratiroidea $(\mathrm{PTH})$ por parte de las glándulas paratiroides, independientemente de sus estimulantes fisiológicos, y representa la primera causa de hipercalcemia en el ámbito ambulatorio. La asociación entre el HPTP y la Hipertensión arterial (HTA) no escapa esta controversia en su aspecto epidemiológico, existiendo aún muy pocos estudios poblacionales explorando este vínculo y su relación con morbimortalidad cardiovascular, dificultando su aceptación plena como causa significativa de HTA secundaria. (p. 1-18)

La paratohormona (PTH), también conocida como hormona paratiroidea, es un poli péptido de una sola cadena de 84 aminoácidos, secretada y almacenada por la glándula paratiroides y uno de los reguladores más importantes del metabolismo mineral. La PTH estimula la síntesis renal de la 1,25-dihidroxi vitamina $D$ y la reabsorción renal de calcio. (Medicina \& Laboratorio, 2017).
La regulación de los niveles de PTH se encuentra controlada por un mecanismo complejo de retroalimentación, en el cual, el calcio iónico, el calcitriol o sus derivados, y el fósforo regulan la secreción de PTH. Sin embargo, se han descrito también otros factores que pueden actuar sobre la glándula paratiroides como los estrógenos, y el factor de crecimiento fibroblástico. (Carrillo, 2010)

La principal función de la paratohormona es mantener la concentración de calcio ionizado en el líquido extracelular. En este sentido Restrepo et al. (2015) señalan que esto se logra a través de los siguientes mecanismos:

1. Estimulación de la resorción ósea por el osteoclasto en forma indirecta y liberación de calcio y fósforo desde el hueso.

2. Estimulación de la reabsorción de calcio e inhibición de la reabsorción del fósforo en los túbulos renales.

3. Estimulación de la producción renal de la 1,25-dihidroxi vitamina D, que aumenta la absorción intestinal de calcio y fósforo.

El hiperparatiroidismo (HPT) es una enfermedad caracterizada por el aumento de secreción de hormona paratiroidea (PTH) a partir de las glándulas paratiroides. Alcaide et al. (2020) describe tres formas clínicas en función de su etiología:

- Hiperparatiroidismo primario: con un aumento de la PTH por la alteración en las propias glándulas paratiroides.

- Hiperparatiroidismo secundario: con elevación de la PTH en respuesta a hipocalcemia producida por otras enfermedades siendo la más frecuente la enfermedad renal.

- Hiperparatiroidismo terciario: que se produce cuando el hiperparatiroidismo secundario se cronifica y funciona de forma autónoma a la causa que lo indujo, aunque ésta se haya corregido. 


\section{NIVELES DE PARATHORMONA RELACIONADO CON EVENTOS CORONARIOS AGUDOS EN PACIENTES CON ENFERMEDAD RENAL CRÓNICA}

Por su parte Martínez (2012) clasifica al hiperparatiroidismo como primario y secundario, y los describe de la siguiente manera:

- Hiperparatiroidismo primario (HP): es una entidad clínica que se caracteriza por la producción autónoma de parathormona (PTH), en la cual hay hipercalcemia o calcio sérico normal-alto, con valores de PTH elevados o inapropiadamente normales.
- Hiperparatiroidismo secundario: Situación clínica en la que las glándulas paratiroides responden adecuadamente a una baja concentración de calcio extracelular (por fallo renal, escasa ingesta, malabsorción, etc.). No obstante, si el aumento de PTH no puede corregir el calcio plasmático, ya sea debido a un trastorno en los órganos responsables del transporte o a la escasez, se desarrolla una hipocalcemia.

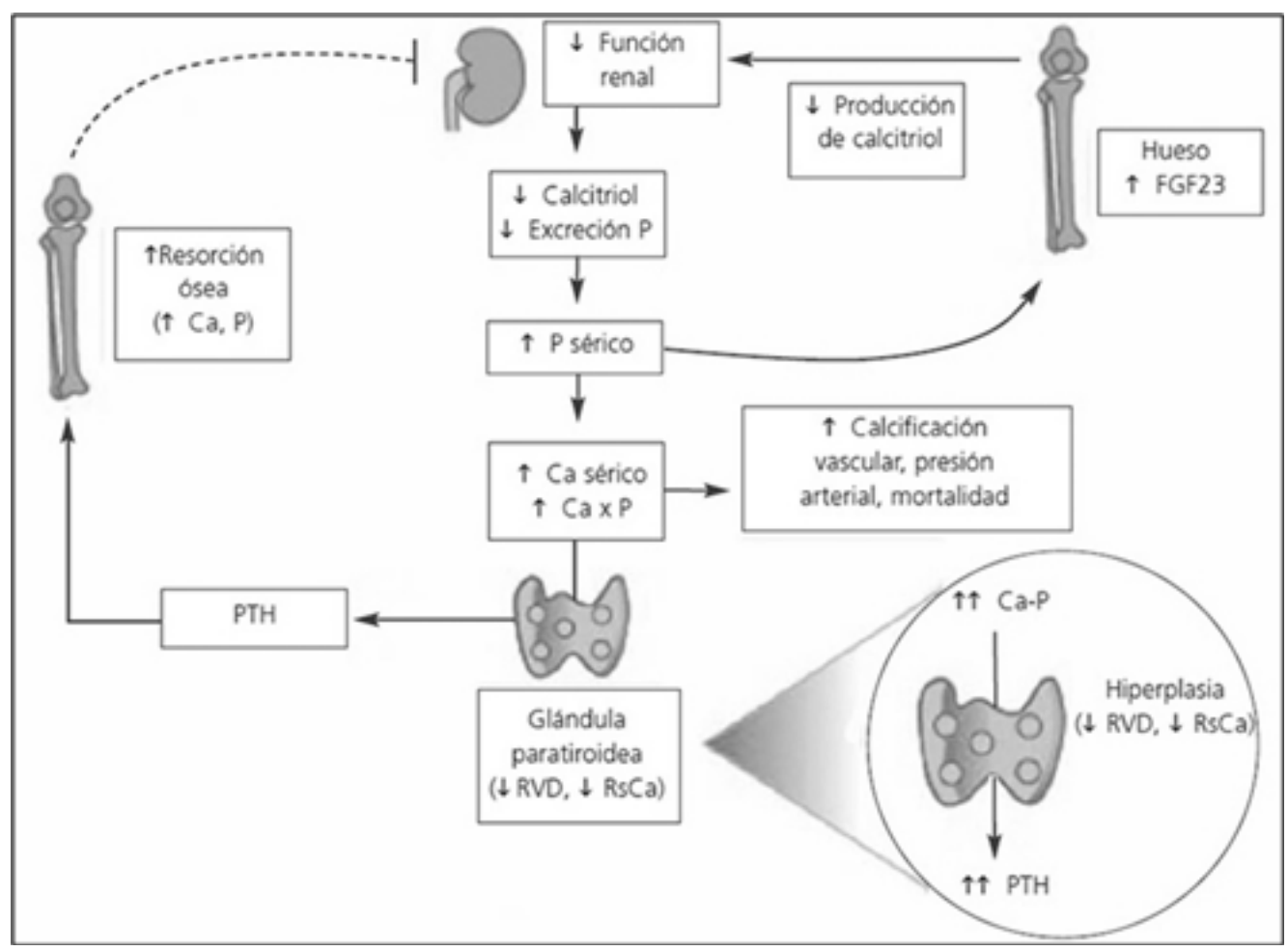

Figura 2. Desarrollo del hiperparatiroidismo secundario en pacientes con enfermedad renal crónica.

Fuente: Activación de los receptores de vitamina $D$ en la optimización del hiperparatiroidismo secundario en diálisis. Herrero et al. (2013). Nefrología 33(4).

Recuperado de: https://scielo.isciii.es/scielo.php?script=sci_arttext\&pi$d=S 0211-69952013000500016$

El hecho de tener niveles de Paratohormona PTH elevados, resulta ser un factor de riesgo importante para la aparición de eventos coronarios agudos, encontrándose significancia estadística entre niveles de $\mathrm{PTH} \geq \mathrm{a} 300 \mathrm{pg} / \mathrm{ml}$ con eventos coronarios agudos, principalmente en el género masculino. (Cediel et al. 2015).
Pese a todo lo anteriormente expuesto, todavía es muy pobre la evidencia en lo que respecta a la relación existente entre los niveles de PTH y el pronóstico una vez la enfermedad coronaria está establecida. (Ramos et al. 2017). 
Estudios recientes han analizado la presencia de condicionantes asociados a un perfil clínico más adverso en aquellos pacientes con angina crónica estable y niveles elevados de PTH. En lo que respecta a las implicaciones pronosticas en pacientes estables, los niveles elevados de PTH también se han relacionado con mayores eventos cardiovasculares adversos. (p.7381).

\section{Conclusión}

En los pacientes con Enfermedad renal crónica (ERC), comparados con la población general, la enfermedad cardiovascular (ECV) es más frecuente y severa. Los pacientes con ERC deben ser vistos como un grupo de alto riesgo cardiovascular, similar a la diabetes y requieren de una atención clínica especial a nivel individual, en el desarrollo de guías clínicas y en el impulso de estudios clínicos específicos a esta población.

La potente asociación causal entre ERC y ECV hace imperativo prevenir el progreso de la ERC, ya que con ello se reduce el riesgo cardiovascular. En los pacientes con ERC la causa de este riesgo cardiovascular elevado es multifactorial, explicada en parte por procesos fisiopatológicos propios.

Existe asociación estadística significativa entre los niveles elevados de parathormona y la incidencia de eventos coronarios, sin embargo, se deben explorar estrategias innovadoras, centradas en los mecanismos fisiopatológicos propios de esta población, sin olvidar que es vital iniciar la prevención en forma precoz y enfocar el tratamiento de estos pacientes de forma multifactorial y bajo un equipo multidisciplinario, así como realizar más estudios prospectivos que permitan ampliar los hallazgos encontrados en la presente investigación.

\section{Bibliografía}

Alcaide, M., Hernandez, E., Reyes, S., Gallart, T., Garcia , J., Sanchez, m., \& Torne, P. (08 de 2020). Hiperparatiroidismo secundario. Actualización y revisión. Cirugia Andaluza, 31(3), 294-299. Recuperado el 30 de 09 de 2021, de https://www.asacirujanos.com/admin/upfiles/revista/2020/Cir_Andal_vol31_n3_11.pdf

Carrillo, N. (2010). Dialnet. Recuperado el 27 de 09 de 2021, de https://dialnet.unirioja.es/servlet/tesis?codigo $=113174$

Cediel, C., Luna , K., Pertuz, A., \& Pérez, L. (12 de 2015). Niveles de parathormona y eventos coronarios agudos en pacientes en hemodiálisis. Biociencias, 10(2), 99-106. Recuperado el 27 de 09 de 2021, de https://dialnet.unirioja.es/descarga/ articulo/5460366.pdf

Clinica Mayo. (03 de 09 de 2021). Mayo Clinic. Recuperado el 29 de 09 de 2021, de https://www.mayoclinic.org/es-es/diseases-conditions/chronic-kidney-disease/symptoms-causes/syc-20354521

Herrero, J., Lopez , J., Maduell, F., Martin, A., Martin, A., Martinez, A., . . . Solozabal, C. (2013). Activación de los receptores de vitamina $D$ en la optimización del hiperparatiroidismo secundario en diálisis. Nefrología, 33(4). doi:https://dx.doi. org/10.3265/Nefrologia.pre2013.May.11901

Martinez, I. (10 de 2012). Hiperparatiroidismo: ¿primario o secundario? Reumatologia Clinica, 8(5), 287-291. doi:DOI: 10.1016/j.reuma.2011.06.001

Medicina \& Laboratorio. (2017). Paratohormona. Medicina \& Laboratorio, 23(1-2), 95-98. Recuperado el 30 de 09 de 2021, de https://www.medigraphic. com/pdfs/medlab/myl-2017/myl171-2g.pdf

Medline Plus. (17 de 12 de 2020). Medline Plus. Recuperado el 28 de 09 de 2021, de https://medlineplus.gov/spanish/pruebas-de-laboratorio/prueba-de-hormona-paratiroidea-pth/

Organización Panamericana de la Salud/Organización Mundial de la Salud (OPS/OMS) . (11 de 03 de 2014). Organización Panamericana de la Salud/Organización Mundial de la Salud (OPS/OMS) . Recuperado el 27 de 09 de 2021, de https://www3. paho.org/hq/index.php?option=com_content\&view $=$ article\&id=9379:2014-kidney-disease-rising-among-seniors-diabetes-hypertension\&Itemi$d=1926 \&$ lang $=$ es

Orozco, R. (03 de 2015). ENFERMEDAD CARDIOVASCULAR (ECV) EN LA ENFERMEDAD RENAL CRÓNICA (ERC). Revista Médica Clínica Las Condes, 26(2), 142-155. doi:https://doi.org/10.1016/j. rmclc.2015.04.003 


\section{NIVELES DE PARATHORMONA RELACIONADO CON EVENTOS CORONARIOS AGUDOS EN PACIENTES CON ENFERMEDAD RENAL CRÓNICA}

Ramos, P., Jaulent, L., Castaneda, M., Martínez, J., Clavel, G., García, L., ... Consuegra, L. (11 de 2017). Hormona paratiroidea, calcidiol, calcitriol y riesgo de eventos adversos en pacientes con síndrome coronario agudo. Medicina Intensiva, 42(2), 73-81. doi:https://doi.org/10.1016/j.medin.2017.09.011

Restrepo, L., Arevalo, J., \& Toro, M. (2015). Metabolismo Mineral y Oseo: Vision general y sus metodos de medicion. Medicina \& Laboratorio, 21(11-12), 511-535. Recuperado el 29 de 09 de 2021, de https://docs.bvsalud.org/biblioref/2018/05/884577/ metabolismo-mineral-y-oseo.pdf

Rojas, J., Olivar, L., Chavez, M., Martínez, M., Wilches, S., Graterol, M., . . . Bermúdez, V. (2017). Hormona paratiroidea, aldosterona e hipertensión arterial ¿una amenaza infravalorada? . Revista Latinoamericana de Hipertensión, 12(1), 1-18. Recuperado el 28 de 09 de 2021, de https://www.redalyc.org/pdf/1702/170250838001.pdf
Serje, E., Guerra, F., \& Pertuz, A. (2014). UTILIDAD DE LA ELEVACIÓN DE PTH INTACTA COMO PREDICTOR DE EVENTOS CARDIOVASCULARES EN PACIENTES NEFRÓPATAS EN HEMODIÁLISIS. Biociencias, 9(1), 15-22. Recuperado el 30 de 09 de 2021, de https://dialnet.unirioja.es/descarga/ articulo/5976568.pdf

Serje, E., Guerra, F., \& Pertuz, A. (06 de 2014). UTILIDAD DE LA ELEVACIÓN DE PTH INTACTA COMO PREDICTOR DE EVENTOS CARDIOVASCULARES EN PACIENTES NEFRÓPATAS EN HEMODIÁLISIS. Biociencias, 9(1), 15-22. Recuperado el 29 de 09 de 2021, de https://dialnet.unirioja.es/descarga/ articulo/5976568.pdf

Sweis, R., \& Jivan, A. (07 de 2020). Manual MSD. Recuperado el 30 de 09 de 2021, de https:// www.msdmanuals.com/es-ve/professional/trastornos-cardiovasculares/enfermedad-coronaria/ generalidades-sobre-los-s\%C3\%ADndromes-coronarios-agudos-sca

\section{CitAR ESTE ARTICULO:}

Lema Tixi, C. E., Padilla Manzano, E. P., \& Salinas Martínez, Ángelo R. (2021). Niveles de Parathormona relacionado con eventos coronarios agudos en pacientes con enfermedad renal crónica. RECIMUNDO, 5(4), 143-151. https:// doi.org/10.26820/recimundo/5.(4).oct.2021.143-151

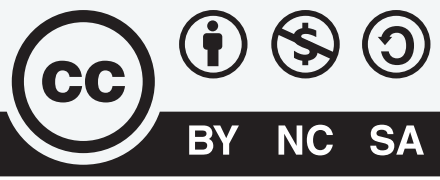

CREATIVE COMMONS RECONOCIMIENTO-NOCOMERCIAL-COMPARTIRIGUAL 4.0. 